\title{
Liebe Leserinnen und Leser, liebe Kolleginnen und Kollegen,
}

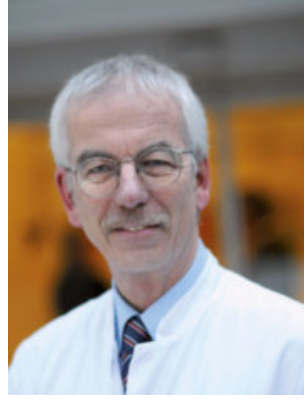

wenn Sie dies lesen, sind wir mitten im meteorologischen Sommer. Im öffentlichen wie auch möglicherweise beruflichen Leben tritt die Haut wieder stärker in den Vordergrund. Wer mehr Haut exponiert, fängt sich auch mehr Probleme ein - Verletzungen, Sonnenbrand, Insektenstiche usw. lösen die Schnieferei in Hausarzt- wie Apothekerberatung ab. Die ZPT hatte sich dem Thema viele Jahre nicht gewidmet. Kein Wunder, dass es Neues zu berichten gilt. Die wissenschaftliche Neuentwicklung eines Birkenrindenextraktes über die EMA-Zulassung bis hin zur Vermarktung in einem Gebiet, wo Phytotherapeutika bislang alles andere als etabliert waren - den Verbrennungswunden - ist eine ausführliche Beschreibung wert (Beitrag Scheffler S. 100). Der beharrlich eingeschlagene Weg sucht Nachahmer! Dass die erste randomisierte Studie hierzu in dem angesehenen Journal PLoS One veröffentlicht wurde, zeigt, dass die internationale wissenschaftliche Öffentlichkeit an Neuigkeiten aus der Phytotherapie interessiert ist.
Die gesunde wie die kranke Haut ist Auf- und Austragungsort für Kosmetika, Medizinprodukte und Arzneimittel. Diese Begriffe befinden sich für den Konsumenten bzw. Patienten allemal, manchmal aber auch für Profis in einer Gemengelage. Wir können Ihnen mit einem qualifizierten Beitrag zur Übersicht verhelfen (Beitrag Latté S. 107). Neurodermitis ist eine Erkrankung, die Naturheilkunde und Komplementärmedizin schon lange beschäftigt. Um phytotherapeutische Externa, die früher viel empfohlen wurden, ist es ruhiger geworden. Es besteht Innovationsbedarf. Eine biologisch sehr interessante, bislang nicht eingesetzte Wirkstoffgruppe aus den Polyphenolen, die Quercetine, wird in einer Pilotstudie untersucht. Die Ergebnisse, die die ZPT als Originalarbeit veröffentlicht, laden zu einer Weiterentwicklung ein (Beitrag Staubach-Renz S. 112).

Sommerzeit ist natürlich auch Gartenzeit! Einer der größten und gepflegtesten der Welt widmet sich neben der sehr gelungenen und jährlich von Hunderttausenden (!) Touristen bestaunten Präsentation seiner Pflanzen auch deren Registrierung, etwa für den Artenschutz und ihrer generellen Beforschung, speziell erfreulicherweise auch der von Heilpflanzen. Das britische Zentrum für botanische Forschung in Kew Gardens in London brachte im Mai 2017 wie jedes Jahr sein von 128 weltweiten Wissenschaftlern extrem sorgfältig recherchiertes Werk „The State of the World's Plants Report 2017“ [1] heraus, darin enthalten das Verzeichnis der „Medically used plants“. In diesem Jahr überraschte es wieder einmal mehr mit der akribischen Aufzählung potenziell und real genutzter Heilpflanzen aus verschiedenen Regionen der Welt. Selbst dem Deutschen Ärzteblatt war dies einen Hinweis wert [2]! Im Einzelnen teilt der Report mit, dass als eher konservative Abschätzung wenigstens 28187 Pflanzenarten derzeit als medizinisch nutzbar gelistet sind. Von denen sind aber nur 4478 (16\%) in medizinischen Periodika erwähnt. Darüber hinaus würden weltweit im Schnitt 15 verschiedene Namen für eine Spezies genutzt. Dies könne durch verstärkte Nutzung etwa der Datenbank Kew's Medicinal Plant Names Service [3] verbessert werden.

Es bleibt weiterhin genügend $\mathrm{zu}$ tun für die Phytotherapie! Helfen Sie uns mit zu einer wissenschaftlich hochrangigen Berichterstattung!

Herzlichst

Ihr

\section{Rainer Stange}

Hinweise

[1] http://www.kew.org/about-our-organisation/ press-media/press-releases/the-state-of-theworlds-plants-report-2017

[2] https://www.aerzteblatt.de/nachrichten/75820

[3] http://www.kew.org/science/data-and-resources/tools-and-services/medicinal-plant-names-services 